\title{
¿Podemos cantar victoria?
}

El martes 19 de agosto -en las instalaciones del Centro de Producción Videográfica de nuestra universidad- se inauguró la "III Jomada por la vida, la justicia y los derechos humanos". Dicha actividad fue organizada en coordinación con el Centro Universitario Cultural, en el marco del décimo segundo aniversario del IDHUCA. El objetivo fundamental que se perseguía con este esfuerzo era el de transmitirle a la población salvadoreña - por la vía del arte, la cultura y la discusión académica responsable- un mensaje claro en relación con la importancia de su participación en la defensa y promoción de los derechos humanos, como elemento indispensable para la construcción de una sociedad realmente democrática.

Así, pues, desde ese día y hasta el sábado 31 de agosto, se mantuvo expuesta al público una colección fotográfica del artista Sebastiao Salgado, dedicada al "Movimiento de los sin tierra" en Brasil. Además, fueron varios los debates realizados alrededor de la problemática desde perspectivas diversas; se llevó a cabo un ciclo de cine-forum así como presentaciones de danza, teatro y música. Para la realización de dichas actividades se contó con las instalaciones de la UCA; pero tarnbién, en esta ocasión, se pudo llegar hasta San Miguel y Santa Ana, a ciertas comunidades de Soyapango, al público "dominguero" de Metrocentro en San Salvador e, incluso, a las personas recluidas en el Centro Penal de Mariona. De esta manera, se le dio continuidad a un evento que año con año — desde 1995- va logrando un mayor impacto en la sociedad salvadoreña.

Para quien no lo sepa, cabe mencionar que el IDHUCA nació en agosto de 1985 por iniciativa y bajo la dirección de Segundo Montes, uno de los seis jesuitas asesinados el 16 de noviembre de 1989. La institución es parte de los bienes que este sacerdote legó al país, en su afán por mejorar la situación de los derechos humanos mediante la superación de las atrocidades sistemáticas que, en ese entonces, tenían lugar en nuestro territorio; diez años antes, en agosto de 1975, el mismo padre Montes había sido pieza clave en la creación del Socorro Jurídico Cristiano.

El surgimiento del Instituto tuvo lugar en un El Salvador distinto al actual. De ello no cabe duda, después de todo lo que ha pasado desde 1985 hasta la fecha: la ofensiva militar insurgente durante noviembre de 1989 y la respuesta gubemamental; el proceso de negociación que arrancó cuando las partes beligerantes se encontraron en Ginebra, allá por abril de 1990; los acuerdos y desacuerdos de esos años; la firma de aquel documento en Chapultepec; la creación y reforma de importantes instituciones relacionadas con el tema de los derechos bumanos; el principio y el fin de la verificación internacional; los acuerdos y desacuerdos de ahora...

Tras todo eso, quien con mala intención o sin ella se atreva a sostener que este nuestro país es el mismo, se encuentra absolutamente equivocado. Porque - para bien o para mal - se ha movido y se encuentra en movimiento. El grave problema que advertimos desde el IDHUCA tiene relación, precisamente, con eso último: que nos encontramos moviéndonos pero en el "filo de la navaja" y no sabemos si ese vaivén nos llevará a una situación mejor o peor que la vivida durante las décadas pasadas, cuando la violencia política y la guerra conmovieron al mundo. Tal inquietud $e$ incertidumbre no es fruto de un ánimo retórico o pesi- 
mista. Lo que en la actualidad está sucediendo dentro del territorio nacional, nos brinda suficientes razones para sostener lo anterior.

Para comenzar a desnudar el arriesgado escenario en el cual nos encontramos inmersos, utilicemos dos instrumentos. El primero: los resultados de la última encuesta realizada por el Instituto Universitario de Opinión Pública (IUDOP), la cual tiene que ver con la situación de violencia que impera dentro del llamado "gran San Salvador". El segundo: el informe del secretario general de la Organización de las Naciones Unidas (ONU), Kofi Anann, de fecha 1 de julio del año en curso.

“La situación en Centroamérica: procedimientos para establecer la paz firme y duradera, y progresos para la configuración de una región de paz, libertad, democracia y desarrollo" es el largo título del documento cuya introducción inicia haciendo memoria de la resolución 51/199, emitida por la Asamblea General de la ONU. En ella, entre otras cosas, se decidió nombrar un funcionario "de alto nivel" para que -mediante visitas periodicas, durante seis meses- pudiese asumir "las responsabilidades de verificación y buenos oficios confiadas a las Naciones Unidas", contando con la asistencia de "una pequeña unidad de apoyo en el terreno". De esa forma forma, le correspondió al conocido y experimentado don Alvaro de Soto seguir involucrado en estos asuntos.

Además de presentar lo que el secretario general considera una "descripción del estado actual de la ejecución de los acuerdos de paz", en este informe se incluye "una evaluación del proceso de paz en El Salvador" que es - según se afirma- la "principal finalidad" del mismo. Para ello, dice el documento, Anann tuvo presentes dos cosas: los objetivos generales establecidos en el acuerdo de Ginebra, firmado el 4 de abril de 1990, y "la aplicación de los mecanismos definidos por las partes para alcanzar esos objetivos en los acuerdos de paz firmados en el Castillo de Chapultepec en México, D.F.", así como en todos los documentos posteriores al 16 de enero de 1992.

¿A qué "objetivos generales" se refiere? Pues a los siguientes: finalizar la guerra por la vía política, en el plazo más corto posible; respeto irrestricto de los derechos humanos; democratización del país; y reunificación de la sociedad. Los mecanismos definidos para el logro de esas ambiciosas pero necesarias metas no fueron pocos y destacan entre ellos -además del establecimiento de una Misión de Observadores de las Naciones Unidas en El Salvador, más conocida como ONUSAL por sus siglas- la formación de la Comisión Nacional para la Consolidación de la Paz (COPAZ) y la reconversión política del Frente Farabundo Martí para la Liberación Nacional (FMLN).

Se impulsaron, además, reformas institucionales dentro de la Fuerza Armada y se creó una Comisión ad hoc para depurarla, además de su drástica reducción numérica. Desaparecieron los antiguos cuerpos de seguridad y la Dirección Nacional de Inteligencia; surgió un nuevo y único cuerpo policial - la Policía Nacional Civil (PNC) - junto a la Academia Nacional de Seguridad Pública, el Consejo Nacional de Seguridad Pública, la Inspectoría General de la PNC y el Organismo de Inteligencia del Estado, instituciones todas bajo la jurisdicción de autoridades civiles. Se disolvieron las entidades paramilitares y se suspendió el reclutamiento forzoso. Para conocer las causas y responsables de los graves hechos de violencia del pasado se creó la Comisión de la Verdad.

Además, se definió como una necesidad la realización de importantes reformas a las instituciones judiciales y a muchas leyes, en cuenta la del Consejo Nacional de la Judicatura. También nació la Escuela de Capacitación Judicial, se creó el Tribunal Supremo Electoral (TSE) y se llevaron a cabo algunas reformas dentro del sistema político. Existió, por un tiempo, el Foro para la Concertación Económica y Social. Finalmente, comenzó a funcionar la Procuraduría para la Defensa de los Derechos Humanos (PDDH).

Pasando revista a esos cuatro grandes objetivos del "proceso de paz salvadoreño" y a los mecanismos diseñados para alcanzarlos, cualquiera descubre de inmediato la inmensa empresa en la que se embarcó la ONU. Ese esfuerzo se realizó, primero, por mandato del Consejo de Seguridad del organismo, desde que se instaló ONUSAL en el terreno el 26 de julio de 1991 hasta el 30 de abril de 1995; de ahí en adelante, la responsabilidad pasó a la Asamblea General y su misión en el país se fue reduciendo gradualmente. Con un enorme aparato inicial para alcanzar sus propósitos de mediación y verificación, la comunidad intemacional intentó llevar a feliz término el cumplimiento de los compromisos establecidos en los acuerdos de paz; ahora - con el informe de Anann- prácticamente ha dado por cerrado el "caso salvadoreño". 
Tras ese descomunal operativo - bueno, mediocre o malo según "el cristal con que se mire", ahora nos encontramos en situación sumamente peligrosa, tal como lo señalamos antes. $Y$ es que está por demás decir que en nuestro país la mayor parte de la gente vive sumida en un ambiente de violencia e inseguridad que - para su desgraciaha alcanzado niveles absolutamente insoportables. El temor generalizado de las personas a ser asesinadas, asaltadas o lesionadas se palpa, se respira, se siente a diario y con sobrada razón.

Al abordar la problemática relacionada con delincuencia, violencia e inseguridad -temas sensibles para quienes presumen con este "proceso salvadoreño"- y publicar los resultados de su última encuesta, el IUDOP ha dado bastante de qué hablar. Quizás la atención mayor se haya centrado en lo que para muchas personas resulta ser la conclusión más impactante: que El Salvador es el país más violento de América Latina. Eso ya lo sabíamos desde antes: lo dijo el Banco Interamericano de Desarrollo (BID) y la Organización Panamericana de la Salud (OPS). Lo que vuelve más interesante la investigación realizada por la institución de la UCA son otros datos puntuales que de ella se desprenden.

Al ser interrogada por el funcionamiento de las instituciones nacionales encargadas de promover el respeto a los derechos humanos y garantizar justicia cuando éstos han sido violados, la población consultada le otorgó el primer sitio a la Procuraduría para la Defensa de los Derechos Humanos (PDDH); en el segundo lugar colocó a la Policía Nacional Civil (PNC). En cambio, la evaluación no resultó tan buena para las dependencias del Órgano Judicial -incluido el sistema penitenciario- y la Fiscalía General de la República; el desempeño de las mismas tendió más a ser calificado como "regular" y "negativo".

Esta última percepción de la gente guarda una estrecha relación con un importante hecho colectivo: en número significativo, las personas no denuncian los actos de violencia que les afectan. ¿Por qué será? Pues, lógicamente, porque las personas "comunes y corrientes" - a partir de su propia experiencia o de otras que conocen- no confian en las autoridades encargadas de investigar los delitos y sancionar a sus responsables. Eso, en palabras más claras y directas, debe entenderse como el predominio de una situación en la cual resultan favorecidas la impunidad o el ejercicio de formas de "justicia privada".
Ante este panorama y al igual que en ocasiones anteriores, altos funcionarios encargados de velar por la tranquilidad y la convivencia social armónica en el territorio nacional —entre ellos el ministro de seguridad pública, Hugo Barrera, y hasta el presidente Armando Calderón Sol- rechazaron enfáticamente la información vertida por el IUDOP, y argumentaron que es una "exageración" y que no debemos ser comparados con otros países.

También, el recién estrenado presidente de la Corte Suprema de Justicia, Eduardo Tenorio, se pronunció sobre el tema en uno de los eventos realizados en la UCA dentro de la "III Jomada por la vida, la justicia y los derechos humanos". Al respecto, dijo que él también se sentía personalmente inseguro pero que, pese a ello, no estaba de acuerdo con los resultados del estudio mencionado pues en El Salvador "las estadísticas son malas". Ricardo Navarro, del Centro Salvadoreño de Tecnología Apropiada (CESTA), al escuchar tal afirmación comentó irónicamente que para nuestra suerte eran "malas" las estadísticas, pues de ser "buenas" sus resultados revelarían una situación mucho más alarmante.

Por encima de esas posturas oficiales, para nuestra reflexión lo que más interesa es la actitud que las personas en nuestra sociedad adoptan ahora frente a la problemática. Llenas de temor y desconfianza, de frustración y desencanto, la mayoría de ellas - como lo revela el estudio del IUDOP_no utiliza una institucionalidad que fue creada o renovada después de largos años de sufrimiento e ilusión: sufrimiento por la violencia política y la guerra; ilusión por la negociación y los compromisos establecidos en los acuerdos de paz.

$Y$ esa actitud popular no es fortuita; tiene suficiente fundamento. La confianza que deposita en la Procuraduría para la Defensa de los Derechos Humanos (PDDH) resulta confirmada plenamente por el secretario general de la Organización de las Naciones Unidas (ONU) en su informe. En el numeral 18 del documento, el señor Annan habla lo que sigue: "Tras un comienzo algo vacilante, se estableció la Oficina de la Procuraduría Nacional (sic) para la Defensa de los Derechos Humanos... Con el correr del tiempo,... ha obtenido un alto nivel de reconocimiento público. Particularmente durante el mandato de su actual titular, ha hecho valientes esfuerzos para cumplir su mandato de supervisar la actuación del Estado y contrarrestar 
los posibles abusos de poder, a pesar de los obstáculos con que ha tropezado a causa de la falta de voluntad política de algunos funcionarios gubernamentales para responder adecuadamente a sus resoluciones y recomendaciones".

En ese marco, el secretario general de la ONU se refiere de manera particular al recorte presupuestario mediante el cual se ha castigado desde el Órgano Ejecutivo la actuación de la señora procuradora para la defensa de los derechos humanos, Victoria Marina Velásquez de Avilés. Eso sucede mientras las organizaciones sociales y la población dentro del país, junto a muchas y prestigiadas instituciones intemacionales, reconocen y premian su meritoria labor.

Sin "pelos en la lengua", el señor Anann lamenta "que la futura consolidación de la Oficina de la Procuraduría Nacional (sic), institución indispensable para el fortalecimiento democrático del Estado y de la sociedad, pueda verse en peligro por el hecho de que su presupuesto para el año actual disminuyera considerablemente en lugar de aumentar, como lo había recomendado mi predecesor". Seguramente con esa misma claridad, el secretario general habría reclamado y hecho un llamado de atención más severo, de haberse conocido los rumores bastante sólidos que circularon -después de la publicación de su informe- sobre una nueva reducción presupuestaria para 1998.

De los aciertos y errores de la Policía Nacional Civil (PNC), es bastante lo que el IDHUCA ha comentado en este y otros espacios, no sólo durante los últimos meses sino desde el mismo nacimiento de la corporación policial. Ahora utilizaremos lo expresado por el secretario general de la ONU para evidenciar las "luces" y "sombras" que existen en el cuerpo y que son percibidas por la población; ese juicio también sirve para explicar, en parte, las razones que animan al público consultado para ubicarla en el segundo sitio de la encuesta, cuando el IUDOP evalúa "las instituciones relacionadas con la justicia y la seguridad pública".

Dice el señor Anann: "A cinco años del comienzo de esta profunda reforma de las estructuras de seguridad pública del país, es evidente que se ha producido una transformación. La Policía Nacional Civil representa un cambio cualitativo de la estructura institucional del país sin precedentes en su historia... Sin embargo, aunque la Policía Na- cional Civil es un órgano de seguridad pública profesional con posibilidades de ejecutar políticas de seguridad pública modemas y democráticas, el modelo establecido por los acuerdos de paz no se ha consolidado sin que se produjeran distorsiones".

Haciendo referencia a las dificultades que desde un principio existieron para el desarrollo del cuerpo, entre ellos el clima de violencia persistente y en aumento, el sectetario general cuestiona diplomáticamente la gestión de las autoridades del Estado encargadas de la seguridad pública por su actuación "no siempre caracterizada por una adhesión firme al fortalecimiento de la nueva fuerza de policía". Así, "se tomaron atajos que se situaron peligrosamente cerca de las prácticas del pasado" y se "alentó la investigación de delitos por grupos situados al margen de la estructura institucional de la Policía Nacional Civil, en tanto que el Departamento para la Investigación del Crimen Organizado -una de las iniciativas más prometedoras de los esfuerzos de la Policía Nacional Civil-se vio considerablemente debilitado".

Además, Anann criticó la legislación de emergencia aprobada para "combatir la delincuencia" y la incapacidad de los mecanismos para controlar el funcionamiento de la institución, entre ellos con sobrada razón- al inspector general de la Policía Nacinal Civil (PNC). Sobre éstos, se expresó así: "nunca lograron establecerse como garantes de la transparencia de los procedimientos policiales ni de la aplicación de una disciplina apropiada". De forma clara, sentencio: "Su labor carece de una coordinación eficaz, no sólo entre sí sino con otras entidades del Estado".

Destaca en su análisis como algo positivo la creación del Consejo Nacional de Seguridad Pública y la posibilidad de que lleve a la práctica "importantes proyectos". Pero, por otra parte, lamentó "que las autoridades del sector estén tratando de obtener la aprobación de la Asamblea Legislativa para modificar alguna de las normas fundamentales del funcionamiento de la Policía Nacional Civil y la Academia Nacional de Seguridad pública sin haber efectuado los necesarios estudios técnicos ni haber analizado las consecuencias de dichas modificaciones".

En otro campo, tras mencionar las reformas que debían introducirse en las leyes y las instituciones encargadas de impartir justicia como resul- 
tado de los acuerdos de paz, el secretario general de la ONU ofrece en su informe una larga y preocupante lista. En ella se incluyen muchas de las deficiencias que aún nos impiden estar satisfechos en este importante rubro y que por lógica - no contribuyen a elevar los niveles de confianza entre la población ni la animan a denunciar los delitos de los cuales son víctimas.

Cuando menciona los "considerables progresos alcanzados" en esta materia, el señor Anann no encuentra más que un ejemplo: la elección de la Corte Suprema de Justicia en 1994. Eso es sumamente desalentador, si se toman en cuenta los siguientes elementos: ineficacia de la justicia constitucional, lentitud de la justicia penal, situación de las y los reclusos que esperan sentencia, infrahumanas condiciones carcelarias, concentración de funciones administrativas en la Corte, inexistencia real de una carrera judicial capaz de fortalecer y estimular a sus miembros, resistencia de otros sectores gubemamentales para respetar las decisiones judiciales...

“Además de estas dificultades - sostiene Anann-, la mayor deficiencia de esta esfera es la ineficacia del proceso de depuración de jueces y funcionarios deshonestos, incompetentes o poco motivados. La Corte Suprema de Justicia y el Consejo Nacional de la Judicatura han evaluado a los jueces de una manera y a un ritmo que no han sido suficientes, habida cuenta de la gravedad de la situación. El que no se hayan hecho mayores progresos al respecto impide cumplir con una condición indispensable para estructurar un sistema que, aunado a una policía eficaz, sea capaz de eliminar la impunidad y garantizar la justicia".

Solamente con lo anterior, el juicio resulta categórico y preocupante; más contundente y grave sería, si el secretario general de la ONU —antes de elaborar el documento que nos ocupa- hubiese recibido la información sobre la "calidad" de al menos uno de los magistrados que acaban de ingresar al máximo tribunal y de la manera cómo llegó a ocupar el cargo.

Sumadas, pues, la encuesta del IUDOP y la evaluación del señor Anann sobre la situación en que se encuentran las instituciones encargadas de investigar los delitos, sancionar a sus responsables

y garantizar el respeto a los derechos humanos de la población, junto a los datos proporcionados por el BID y la OPS en relación con lo que está sucediendo en El Salvador, el panorama no es tan brillante. Ante él, las y los funcionarios gubernamentales - lejos de adoptar posiciones de rechazodeberían aprovechar tales herramientas serias y profesionales para revisar lo actuado y diseñar políticas adecuadas, a fin de encontrar solución a los problemas. No obstante la cita que incluimos anteriormente, hay que considerar lo que en otro momento dijo el presidente de la Corte Suprema de Justicia: "aceptada la enfermedad, podemos pasar al ensayo de ideas que pueden traducirse en una terapia eficaz".

Esa es una gran verdad que debe traducirse en hechos concretos, pues el primer paso para comenzar a salir de esta grave y compleja problemática es conocer y reconocer que en materia de seguridad pública, justicia y respeto a los derechos humanos estamos bastante mal; que esa es la realidad que salta a la vista y que la inmensa mayoría de población la está viviendo y sufriendo en came propia. Incluso, hasta familiares de algunas altas autoridades gubernamentales han sido víctimas de este flagelo. Entonces, ¿por qué esa insistencia en vender al exterior una imagen falsa de nuestro país, cuando día a día nos golpea en el rostro un clima generalizado de violencia, inseguridad y temor?, ¿por qué no se acepta de una vez por todas esta situación y se proponen las medidas más acertadas para enfrentarla y reducirla?

El secretario Anann hizo varias recomendaciones en ese sentido. Veamos algunas: que el "mo- 
delo democrático de seguridad pública establecido en los acuerdos de paz" se convierta en política nacional; que el Consejo Nacional de Seguridad Pública "acoja favorablemente las iniciativas, criticas y propuestas de los miembros de la sociedad civil y sus organizaciones; que el Estado invierta recursos para el desarrollo de la PDDH y que la institución se esfuerce para lograr tener "alcance nacional"; que el Órgano Judicial de muestras de "su determinación a implantar los cambios necesarios cuanto antes", teniendo como especial prioridad la "depuración de jueces y funcionarios corruptos o ineficientes".

En otros aspectos, también propuso algunas cosas: que "llegue a feliz término" el Programa de Transferencia de Tierras y que se cuente con la cooperación del Instituto Salvadoreño de Transformación Agraria para la transferencia de las propiedades que superan el límite constitucional de 245 hectáreas; que, con flexibilidad y originalidad, se den progresos tangibles en lo relativo a la transferencia de los asentamientos humanos rurales; que rápidamente se hagan los "ajustes necesarios" para superar "las dificultades con que han tropezado los beneficiarios potenciales del Fondo de Protección para Lisiados y Discapacitados"; y que se aprueben y apliquen "las reformas electorales propuestas por la Comisión Multipartidaria designada por el presidente Calderón Sol".

Además, finalizó con una importante recomendación en los siguientes términos: "quisiera expresar mi esperanza de que el pueblo salvadoreño no olvide la trágica experiencia del pasado. Sólo aquéllos capaces de reconocer y encarar un pasado terrible pueden aprender la lección y asegurarse de que nunca jamás se repita al avanzar hacia la creación de un nuevo futuro para las generaciones venideras". En tal sentido, instó a la aprobación de las "leyes que den a las víctimas de violaciones a sus derechos humanos y sus familiares una indemnización justa por sus sufrimientos". En aras de "contribuir a la formación de una memoria colectiva en que marchen de la mano el reconocimiento del valor de la paz y el respeto a la dignidad humana, debería considerarse la posibilidad de enseñar a los niños de las escuelas la historia de los años de conflicto como parte del currículo nacional de educación, en el cual se incluiría el informe de la Comisión de la Verdad como obra de consulta de importancia incuestionable para la historia de El Salvador".
Esta preocupación no es vana; tiene su origen en un hecho: para el señor Anann, al igual que para muchas personas y sectores de la sociedad salvadoreña, "las recomendaciones relativas a la reconciliación fueron de oídas". "En resumen dijo al respecto el secretario general- es inevitable hacer una valoración poco positiva de las medidas adoptadas en relación a las recomendaciones más importantes de la Comisión de la Verdad... Es realmente desalentador que no se haya aprovechado la oportunidad singular que representaba la Comisión y su labor para alcanzar progresos importantes en la eliminación de la impunidad y el fomento de un clima de reconciliación nacional".

Así, pues, en este El Salvador que ya está por cumplir seis años sin guerra, las cosas están como están. Mientras, por ejemplo, existe un Consejo de Seguridad Pública, la gente se siente -con sobrada razón- cada vez más insegura, a consecuencia de los elevados índices de violencia y criminalidad en el país. Mientras hay nuevas y reformadas instituciones, la gente no confia en ellas y no las utiliza en favor del respeto a sus derechos humanos. Mientras se habla de un "país políticamente vibrante" en el marco de una "nueva correlación de fuerzas", la mayoría de los partidos no da muestras de ser realmente responsable y seria de cara a las necesidades más urgentes de la población. Mientras se promocionan cifras macroeconómicas saludables, la salud y la economía de una inmensa cantidad de gente se encuentra en franco deterioro al igual que su situación de empleo, educación, vivienda, cultura, recreación y medio ambiente.

Es en ese El Salvador actual y dentro del estado en que se encuentra el respeto a los derechos humanos de sus habitantes -lleno de luces y sombras, logros y fracasos, expectativas y desencantos, aspiraciones y frustraciones, contrastes insultantes a la dignidad de las personas, discursos en otros tonos y lastres no superados - donde nos corresponde actuar. Siendo integrantes de una institución de la sociedad que trabaja en la defensa y promoción de la dignidad humana, es ese el lugar y el momento donde nos encontramos; ahí es donde debemos tener la suficiente capacidad para ubicar los retos del momento y las estrategias más adecuadas que nos permitan enfrentarlos con eficacia.

Así, desde nuestra perspectiva, el desafio más importante de esta etapa $-y$ por tanto ineludible- es el de contribuir a incentivar la participa- 
ción -consciente, activa - de la población en la protección y ampliación de sus derechos y libertades fundamentales, mediante el uso adecuado y oportuno de las instituciones correspondientes, tanto del Estado como de la sociedad. En otras palabras, ahora que la comunidad internacional tiene la mirada puesta en realidades que no son la salvadoreña, a nosotras y nosotros nos toca buscar y lograr que el sistema nacional de protección de los derechos humanos funcione real e integralmente, en conjunto y desde cada uno de sus componentes: las instituciones del Estado, las expresiones organizadas de la sociedad y la población que - a final de cuentas - debe usarlas y beneficiarse de ello.

Para eso, debemos encontrar formas creativas e inteligentes que - por un lado- modifiquen de raíz la visión distorsionada y peligrosa que actualmente prevalece en el país en relación con los derechos humanos. Esa percepción negativa y restringida, incorrecta y polarizante, debe ser superada lo más pronto posible. Por otro lado, es necesario diseñar los mecanismos y las modalidades para que la gente vaya adquiriendo confianza y se involucte en la solución racional, civilizada y pacífica de sus conflictos.

Transcurridos veintidós años desde el surgimiento del Socorro Jurídico y doce del IDHUCA, también resulta oportuno destacar la importancia que en la actualidad continúan teniendo los organismos de la sociedad que trabajan en este campo, debido a que $\longrightarrow$ pese a que ya pasaron cinco años desde el fin de la guerra- siguen siendo muchas y muy graves las carencias en la materia. No es un secreto que la mayoría de las personas en El Salvador - sobre todo sus niñas y sus niños- es víc- tima de la injusticia estructural que predomina, quizás más dura y difícil que antes como resultado de la confrontación armada y los efectos del actual modelo económico, basado en una desigual competencia que sólo genera mayores niveles de exclusión.

Además -independientemente de que hayan terminado los combates militares-, entre la gente persiste el temor y la inseguridad pues aún no se han superado otras formas de violencia surgidas de esa misma injusticia estructural, pero que también tienen bastante relación con la impunidad y los efectos sicológicos derivados de lo que muchas personas tuvieron que pasar. Colocados en medio de esa situación y después de todo lo que hemos vivido y sufrido de 1975 en adelante, ahora la razón principal de quienes trabajamos por la vida, la justicia y los derechos humanos es una y muy grande: contribuir a mantener en alto la certeza de que es posible construir una sociedad diferente.

De ahí el sentido de todas las actividades que se desarrollaron a lo largo de estos días de agosto, en el marco de la "IIl Jornada": el de una invitación para que seamos cada vez más las personas que nos involucremos, de lleno, en la hermosa e impostergable tarea de promover los valores más elevados de la humanidad. Y es que, tal como sentenció el padre Montes al final de su último escrito publicado, en El Salvador: "No es tiempo todavía de cantar victoria por la vigencia de los derechos humanos, pero tampoco es tiempo aún para la desesperanza".

Instituto de Derechos Humanos de la Universidad Centroamericana "José Simeón Cañas" 